\title{
SELECTION OF THE WIDTH OF A CAISSON
}

\author{
Chi Chao David Tung \\ Department of Civil Engineering, North Carolina State University, Raleigh, North Carolina, U.S.A., tung@ncsu.edu \\ Jaw Guei Lin \\ Department of Harbor and River Engineering, National Taiwan Ocean University, Keelung, Taiwan, R.O.C.
}

Follow this and additional works at: https://jmstt.ntou.edu.tw/journal

Part of the Civil and Environmental Engineering Commons

\section{Recommended Citation}

Tung, Chi Chao David and Lin, Jaw Guei (2011) "SELECTION OF THE WIDTH OF A CAISSON," Journal of Marine Science and Technology. Vol. 19: Iss. 1, Article 7.

DOI: 10.51400/2709-6998.2137

Available at: https://jmstt.ntou.edu.tw/journal/vol19/iss1/7

This Research Article is brought to you for free and open access by Journal of Marine Science and Technology. It has been accepted for inclusion in Journal of Marine Science and Technology by an authorized editor of Journal of Marine Science and Technology. 


\section{SELECTION OF THE WIDTH OF A CAISSON}

Acknowledgements

The first 5 sections of this paper are taken from reference 5 . 


\title{
SELECTION OF THE WIDTH OF A CAISSON
}

\author{
Chi Chao David Tung* and Jaw Guei Lin**
}

Key words: port design, caisson, probability.

\begin{abstract}
This study is concerned with the selection of the width of a caisson. At present, the width is determined based on the consideration that, struck by a prescribed design impulsive breaking wave force, a caisson must remain stable against sliding and rocking. In this study, the problem is formulated in a different way. The equations of equilibrium of a caisson, together with the criteria for the caisson to remain at rest, are used to derive expressions for the selection of the width. The criteria of a rest mode are presented graphically using the applied force as the abscissa and the coefficient of friction between the caisson and the base as the ordinate. The region corresponding to a rest mode is indicated. The present approach gives results that are equivalent to those of the existing approach and, by treating the coefficient of friction and the applied force as random, the safety of a caisson is given in probability terms.
\end{abstract}

\section{INTRODUCTION}

At present, the width of a caisson is selected based on the consideration that, struck by a prescribed impulsive breaking wave force, a caisson remains stable against sliding and rocking [3]. Thus, the resultant force representing the caisson's resistance against sliding is made larger than that which causes it to slide, and the resultant moment that counters the rocking tendency of the caisson is made larger than that which causes it to rock. To account for uncertainties, safety factors are applied to the two conditions.

In the present study, the equations of equilibrium of a caisson, together with the criteria for a rest mode are used to derive the expressions of the conditions for a rest mode. These expressions are presented in graphical form with the nondimensionalized breaking wave force as the abscissa and the coefficient of friction of the limiting Coulomb friction force between the caisson and the base as the ordinate. The region

Paper submitted 09/07/09; received 01/06/10; accepted 01/10/10. Author for correspondence: Chi Chao Tung (e-mail: tung@ncsu.edu).

*Department of Civil Engineering, North Carolina State University, Raleigh, North Carolina, U.S.A.

**Department of Harbor and River Engineering, National Taiwan Ocean University, Keelung, Taiwan, R.O.C. corresponding to a rest mode is identified. The graph shows at a glance whether a caisson is in a rest mode or not. The two expressions of the width of the caisson, each for an appropriate range of values of the applied impulsive force, are derived. It is shown that the two expressions are equivalent to the two conditions used in the existing method [3]. Two examples are given to show how the appropriate width of a caisson is chosen and what the corresponding safety factors against sliding and rocking are. Considering the applied force and the coefficient of friction as random, the probability that a caisson remains at rest is determined.

The model of the caisson considered in this study is the same as that in Example 4.1 in section 4.2 in [3] and the model of wave pressures is taken from section 4.2 of the same reference. For the sake of easy reference, these models are repeated here.

\section{MODELS AND CONDITIONS OF REST MODE}

\section{Model of Caisson}

A caisson is modeled as a rigid body, rectangular in elevation and its footprint is of unit length as shown in Fig. 1. The width and height of the caisson are respectively $B$ and $H$. The design water level (including tide level) is shown. The part of the caisson above the design water level is made of concrete whose unit weight is $\gamma_{C}=2.4 \mathrm{t} / \mathrm{m}^{3}$. The part of the caisson below the design water level consists of concrete compartments filled with sand. It is assumed (arbitrarily) that half of that part of the caisson is concrete and half of it is sand whose unit weight is $\gamma^{\prime}=1.1 \mathrm{t} / \mathrm{m}^{3}$. The coefficient of friction is $\mu=0.6$. The values of $h, d, h^{\prime}, h_{C}, H$ and $D$ used in the examples are given in the parentheses in the figure.

\section{Model of Wave Force}

The distributions of wave pressures are shown in Fig. 2. To use the formulas and graphs given in [3], the quantities to be specified are: $H_{O}$, the deep water wave height, $T_{1 / 3}$, the significant wave period and $\beta=15^{\circ}$, the angle between the direction of wave approach and the normal of the vertical wall, and $i=1 / 100$, the sea bottom slope (see Fig. 1).

\section{Equilibrium Equations and Criteria for a Rrest Mode}

Referring to Fig. 3, the free-body diagram of a caisson, the resultant of the wave pressures on the vertical wall of the caisson is the force $P$ and the resultant of the uplift pressures is 


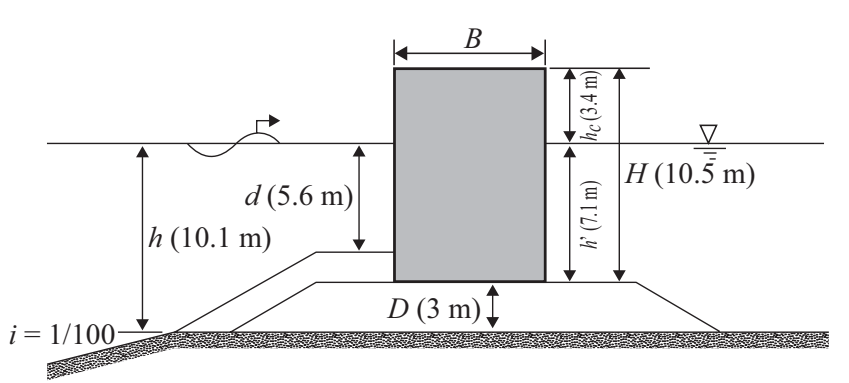

Fig. 1. Caisson.

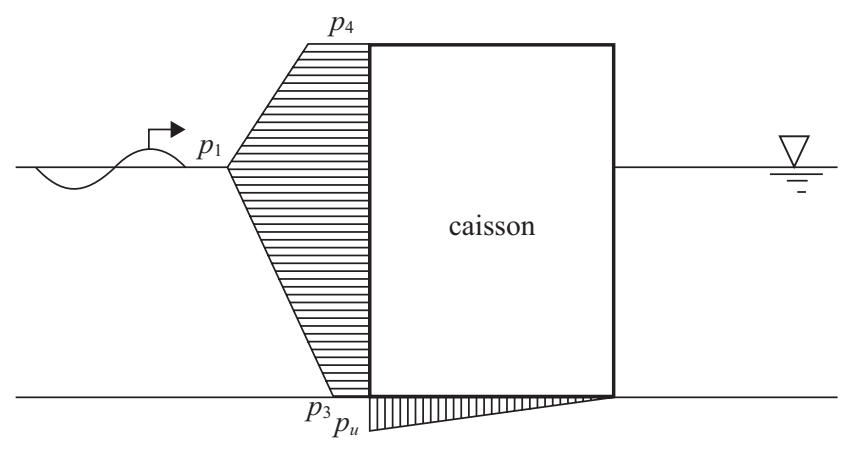

Fig. 2. Wave pressures.

$U$. A caisson, initially at rest, under the action of $P$ and $U$, may be initiated into many modes of motion. It may remain at rest, slide, rock, and slide and rock simultaneously. In Fig. 3, $C$ is the center of mass of the caisson, $W$ is its weight in water. The vertical reaction force is $f_{y}$ whose line of action is at a distance $\xi$ from $C$ and $f_{x}$ is the horizontal reaction force. The force $P$, for convenience, is expressed in terms of $W$ as $P=W k$ where $k$ is a dimensionless quantity. The uplift force $U$ is expressed in terms of $P$ as $U=P q=W k q$ where $q$ is a dimensionless quantity.

In the short duration $\Delta t$ of action of the impulsive forces, the equations of equilibrium are:

$$
\begin{gathered}
f_{x}=-P=-W k \\
f_{y}=W-U=W(1-q k)
\end{gathered}
$$

and, by taking moment of the forces about $C$,

$$
f_{x} H+f_{y} \xi-P\left(H_{P}-H_{C}\right)-U B / 6=0
$$

where $H_{P}$ is the distance between the line of action of the force $P$ and the caisson's base and $H_{C}$ is the distance between the center of mass $C$ and the base as shown in Fig. 3 .

From (1), (2) and (3), we have

$$
\xi=-\frac{P H_{P}+(B U / 6)}{W-U}=-\frac{B k\left[\left(H_{P} / B\right)+(q / 6)\right]}{1-q k}
$$

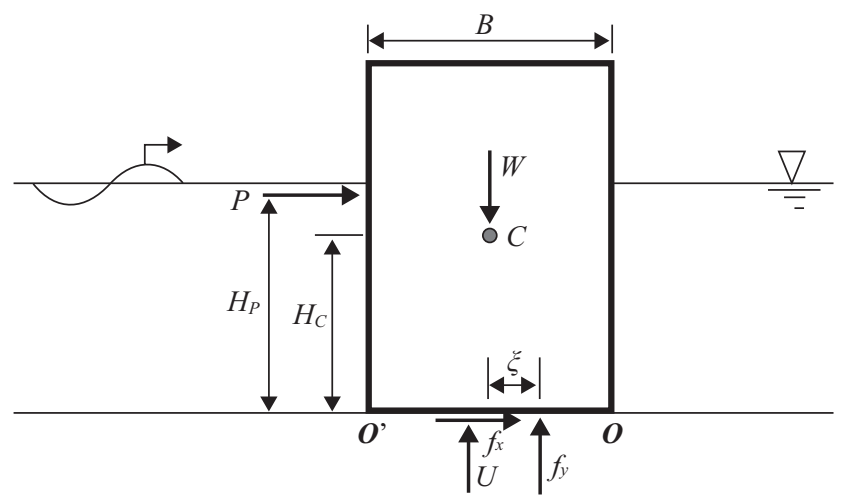

Fig. 3. Free body diagram of caisson.

For the body to be in contact with the base, $f_{y}$ must not be less than zero. From (2), this requires

$$
k \leq \frac{1}{q}
$$

For the caisson to be at rest, $\left|f_{x}\right|$ must not exceed the limiting Coulomb friction force $\mu f_{y}$. That is,

$$
\mu \geq \frac{\left|f_{x}\right|}{f_{y}}=\frac{k}{1-q k} \equiv \mu_{0}(k)
$$

Finally, $f_{y}$ must remain within the base $O O^{\prime}$ of the caisson. That is, $|\xi| \leq B / 2$. From (4), this condition is

$$
k \leq \frac{1}{\left(2 H_{P} / B\right)+(4 q / 3)} \equiv k_{A}
$$

It may be verified that $k_{A} \leq 1 / q$. The conditions (6) and (7) constitute the criteria for the caisson to be at rest. These conditions are presented graphically in Fig. 4 using the parameters $k$ and $\mu$ as the horizontal and vertical axes respectively. The curve $O A$, (or $\left.\mu_{0}\right)$ and the line $A H$, (or $\left.k=k_{A}\right) k$ intersect at $A$, whose coordinates are $k=k_{A}$ and

$$
\mu=\frac{k_{A}}{1-q k_{A}}=\frac{1}{\left(2 H_{P} / B\right)+(q / 3)} \equiv \mu_{A}\left(>k_{A}\right)
$$

The shaded region in Fig. 4 represents the rest mode. That is, a caisson is at rest provided the values of $k$ (the normalized force) and $\mu$ (the coefficient of friction) correspond to a point that lies in the shaded area in Fig. 4.

\section{DETERMINATION OF CAISSON WIDTH}

Noting that $k=P / W, q=U / P, U=p_{u} B / 2$ where $p_{u}$ is the pressure of uplift force at the toe of the caisson (see Fig. 3), 


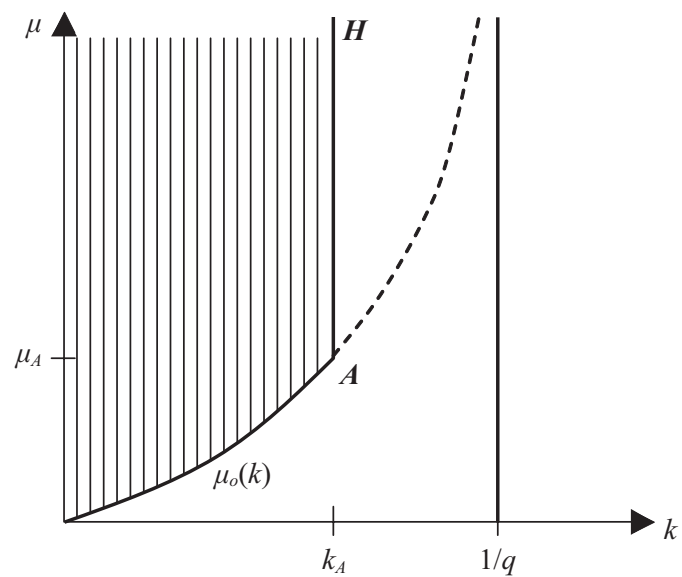

Fig. 4. Region of rest mode in $k-\mu$ plane.

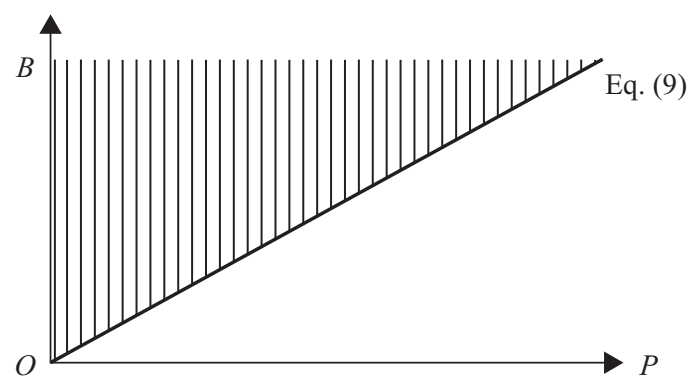

Fig. 5. Region of $B$ that satisfies (9).

and by expressing $W$ in terms of the effective unit weight $\gamma_{e}\left(W=H B \gamma_{e}\right)$ of the caisson in water, the condition (6) requires

$$
B \geq \frac{P}{\mu\left[H \gamma_{e}-\left(p_{u} / 2\right)\right]}
$$

A sketch of $B$ as a function of $P$ is given in Fig. 5. The condition (6) is satisfied so long as $B$ assumes a value in the shaded region in Fig. 5.

In the same way, the condition $k \leq k_{A}$ in (7) requires

$$
B \geq \sqrt{\frac{2 P H_{P}}{\left[H \gamma_{e}-\left(2 p_{u} / 3\right)\right]}}
$$

The quantity $B$ as a function of $P$ is sketched in Fig. 6 . The condition (7) is satisfied so long as $B$ assumes a value in the shaded region in Fig. 6.

The line in (9) and the curve in (10) intersect at

$$
P \equiv P_{C}=\frac{2 H_{P} \mu^{2}\left[H \gamma_{e}-\left(p_{u} / 2\right)\right]^{2}}{\left[H \gamma_{e}-\left(2 p_{u} / 3\right)\right]}
$$

That is, for $P \leq P_{C}$, the width of a caisson is given by (10) and for $P \geq P_{C}$, it is given by (9). Combining Figs. 5 and 6 , the width of a caisson that satisfies (6) and (7) is given in Fig. 7.

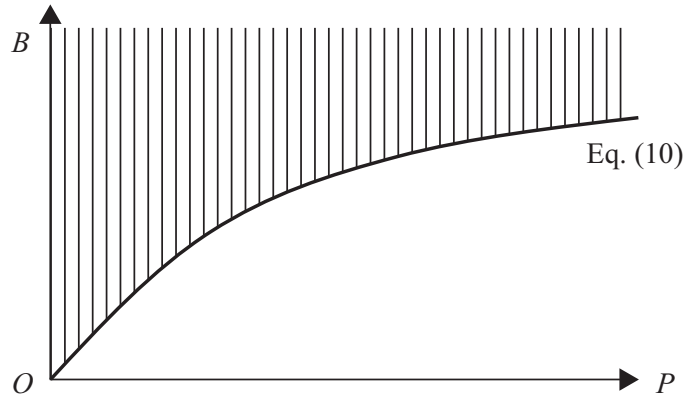

Fig. 6. Region of $B$ that satisfies (10).

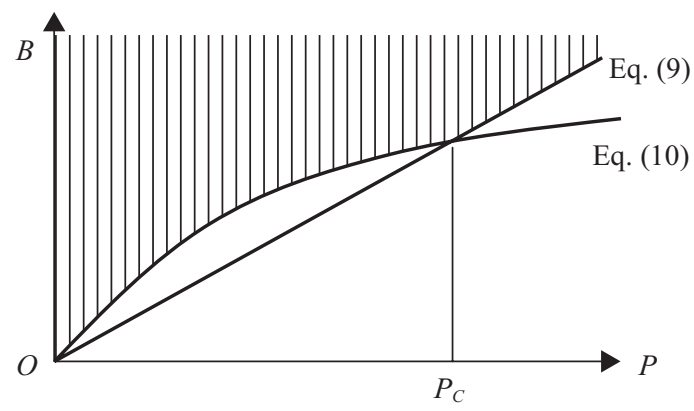

Fig. 7. Region of $B$ that satisfies both (9) and (10).

The condition (6) may be re-written as $\mu \geq \frac{P}{W-U}$, or

$$
\frac{\mu(W-U)}{P} \geq 1
$$

Eq. (12) states that the limiting frictional force must be larger than the force $P$. This is the same as Eq. (4.16) in Goda (Goda, 1985) which ensures that the caisson remains stable against sliding for the fact that in Goda, 1985, a safety factor against sliding is applied.

Similarly, the condition (7) may be written as $P H_{P} \leq$ $(W B / 2)-(2 U B / 3)$ or

$$
\frac{W t-M_{U}}{M_{P}} \geq 1
$$

where $t=B / 2$ and $M_{U}=2 U B / 3$. Here, $M_{U}$ is the moment of $U$ about the heel of the caisson and $M_{p}$ is the moment of $P$ about the base. Eq. (13) is the same as Eq. (4.17) in [3] except Eq. (4.17) in [3] has a safety factor against rocking; satisfaction of (13) ensures safety against rocking.

The following are two examples, each consisting of two cases, given to show how the width of a caisson may be chosen and the interpretations of the caisson's safety in terms of safety factors and in the framework of the present method. 


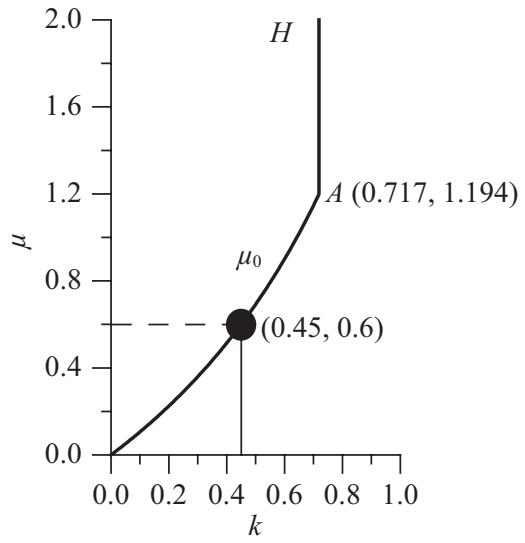

Fig. 8. Diagram showing rest region and the point with coordinates 0.45 and 0.6 in $k-\mu$ plane for Example $1, B=15.83 m$, SF against sliding $=\mathbf{1}, \mathrm{SF}$ against overturning $=\mathbf{2 . 2 7}$.

\section{Example 1}

Consider the caisson in Fig. 1. Using the data given in [3] Example 4.1 except the value of $B$, with $H_{O^{\prime}}=6.3 \mathrm{~m}, T_{1 / 3}=$ $11.4 \mathrm{~s}, \beta=15^{\circ}$, and tide level $0.6 \mathrm{~m}$, we have, $P=94.5 \mathrm{t} / \mathrm{m}$, $M_{P}=487.4 t-m / m, H_{P}=5.158 \mathrm{~m}, p_{U}=6.65 \mathrm{t} / \mathrm{m}, U=3.325 \mathrm{~B}$, $M_{U}=6.65 B^{2} / 3, \gamma_{e}=1.264$, and $P_{C}=42.43 \mathrm{t} / \mathrm{m}(<P=94.5 \mathrm{t} / \mathrm{m})$. Thus, from (9), $B \geq 15.834 \mathrm{~m}$. If the width of the caisson is chosen as $B=15.374 \mathrm{~m}$, then $W=210.15 \mathrm{t} / \mathrm{m}, U=52.64 \mathrm{t} / \mathrm{m}$, $M_{U}=555.75 t-m / m, k=P / W=0.45, q=U / P=0.557, k_{A}=$ $0.719(>k=0.45), \mu_{O}(k)=0.6$ and $\mu_{A}=1.2\left(>\mu_{O}(k)=0.6\right)$.

The point with $k=0.45$ and $\mu_{O}(k)=0.6$ is marked as a heavy dot in Fig. 8. It is noted that the point lies on the curve $\mu_{O}(k)$ indicating that the caisson with width $B=15.834 \mathrm{~m}$ has no safety margin for the caisson to be at rest. The safety factor against sliding is equal to unity according to (12) or equation (14.6) in [3]. The safety factor against rocking is 2.27 , according to Eq. (4.17) in the same reference. That is, the caisson is on the verge of sliding but it has ample margin against rocking.

Using Eq. (4.16) in [3], and setting the safety factor against sliding equal to 1.2 , we have $B=19.0 \mathrm{~m}, W=252.17 \mathrm{t} / \mathrm{m}, U=$ $63.18 \mathrm{t} / \mathrm{m}, M_{U}=880.22 t-m / m, q=0.6685, k=0.375, k_{A}=0.7$ (>0.375), $\mu_{O}(k)=0.5$ and $\mu_{A}=1.3$. The point $(0.375,0.6)$ in the $k-\mu$ plane now lies away from the boundary curve $\mu_{O}(k)$ within the region corresponding to the rest mode as shown in Fig. 9. The safety factor against rocking in this case is 3.27.

\section{Example 2}

In this example, the configuration of the caisson is the same as that in Example 1 except the width $B$. The applied force is chosen to be much smaller than that in Example 1. Thus, with $H_{O^{\prime}}=3 \mathrm{~m}, T_{1 / 3}=7 \mathrm{~s}, \beta=15^{\circ}$, and tide level $0.6 \mathrm{~m}$ we have, in this case, $P=35.8 \mathrm{t} / \mathrm{m}, M_{P}=185.4 t-\mathrm{m} / \mathrm{m}, H_{P}=5.183 \mathrm{~m}, p_{U}=$ $2.64 \mathrm{t} / \mathrm{m}, U=1.32 \mathrm{~B}, M_{U}=2.46 \mathrm{~B}^{2} / 3, \gamma_{e}=1.264$, and $P_{C}=46.7$ $\mathrm{t} / \mathrm{m}(>P=35.8 \mathrm{t} / \mathrm{m})$, Thus, from $(10), B \geq 5.677 \mathrm{~m}$. With $B=$ $5.677 \mathrm{~m}$, we have, $W=75.35 \mathrm{t} / \mathrm{m}, U=7.5 \mathrm{t} / \mathrm{m}, M_{U}=28.4$

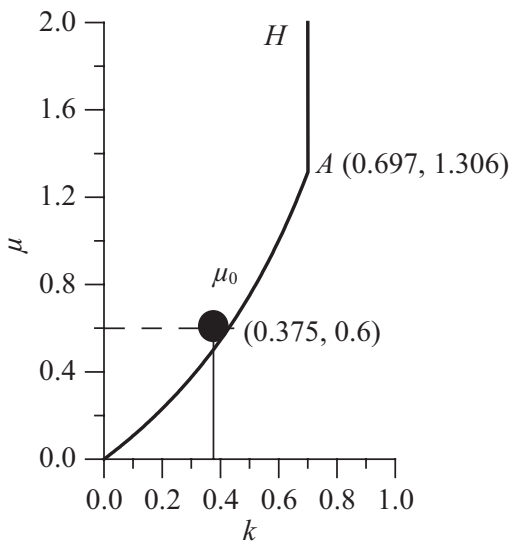

Fig. 9. Diagram showing rest region and the point with coordinates 0.375 and 0.6 in $k-\mu$ plane for Example $1, B=19 m$, SF against sliding = 1.2, $\mathrm{SF}$ against overturning $=3.27$.

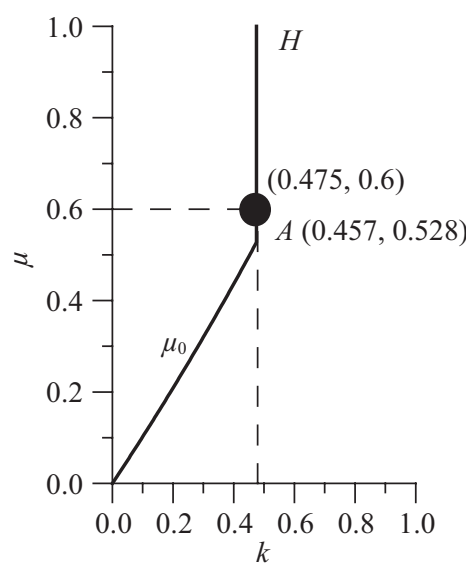

Fig. 10. Diagram showing rest region and the point with coordinates 0.475 and 0.6 in $k-\mu$ plane for Example $2, B=5.677 \mathrm{~m}$, SF against sliding $=1.14, \mathrm{SF}$ against overturning $=1$.

$t-m / m, k=P / W=0.475, q=U / P=0.209, k_{A}=0.475(=k)$, $\mu_{O}(k)=0.527$ and $\mu_{A}=0.527\left(=\mu_{O}(k)\right)$. The point with $k=$ 0.475 and $\mu=0.6$ is shown in Fig. 10. It is seen that the point lies on the boundary $A H$ of a rest mode. The safety factor against rocking is equal to unity. The safety factor against sliding is $1.14(<1.2)$.

By setting the safety factor against rocking equal to 1.2, we have, according to $\mathrm{Eq}(4.17)$ in [3], $B=6.217 \mathrm{~m}, W=82.51 \mathrm{t} / \mathrm{m}$, $U=8.21 \mathrm{t} / \mathrm{m}, M_{U}=34.0 t-m / m, q=0.229, k=0.434, k_{A}=$ $0.508(>k=0.434), \mu_{O}(k)=0.487$ and $\mu_{A}=0.575$. The point $(0.434,0.6)$ in the $k-\mu$ plane lies within the region of rest mode as shown in Fig. 11. The safety factor against sliding is 1.245 (> 1.2).

\section{TREATING $K$ AND $\mu$ AS RANDOM VARIABLES}

Since the values of the forcing function and the coefficient of friction can not be stated with certainty, they are treated as 


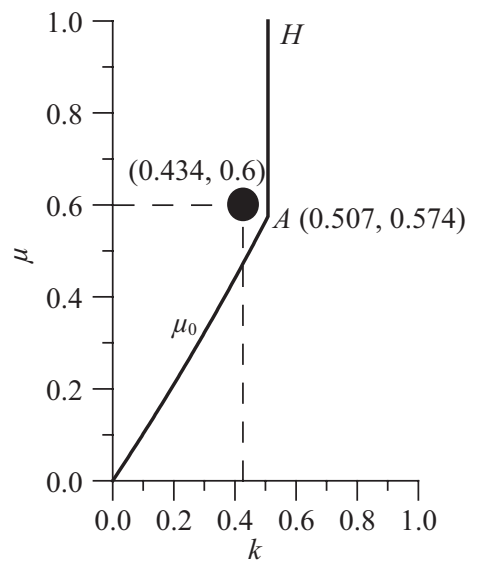

Fig. 11. Diagram showing rest region and the point with coordinates 0.434 and 0.6 in $k-\mu$ plane for Example $2, B=6.217 m$, SF against sliding $=1.245, \mathrm{SF}$ against overturning $=1.2$.

random variables so that the safety of a caisson against a breaking wave force may be assessed in terms of probability.

The random variables $k$ and $\mu$ are naturally statistically independent. In this study, $k$ is considered a Gumbel random variable and $\mu$ is a Lognormal random variable. That is, the probability density function of $k$ is [2]

$$
\begin{aligned}
f_{k}(x)= & \alpha_{k} \exp \left[-\alpha_{k}\left(x-u_{k}\right)-\exp \left(-\alpha_{k}\left(x-u_{k}\right)\right)\right], \\
& -\infty<\mathrm{x}<\infty
\end{aligned}
$$

The parameters $\alpha_{k}$ and $u_{k}$ are: $\alpha_{k}=\pi / 6 \sigma_{k}$ and $u_{k}=E[k]-$ $(0.577) / \alpha_{k}$. Here and hereafter, $E[]$ is the expected value of the quantity enclosed in the brackets and $\sigma$ is the standard deviation of the random variable in the subscript.

As a lognormal random variable, the probability density function of $\mu$ is [2]

$$
f_{\mu}(y)=\frac{1}{\sqrt{2 \pi} \varsigma_{\mu} y} \exp \left[-\frac{1}{2}\left(\frac{\ln y-\lambda_{\mu}}{\varsigma_{\mu}}\right)^{2}\right] \quad 0<\mathrm{y}<\infty
$$

The parameters $\varsigma_{\mu}$ and $\lambda_{\mu}$ are: $\lambda_{\mu}=E[\mu]-\varsigma_{\mu}^{2} / 2, \varsigma_{\mu}^{2}=\ln \left(1+k_{\mu}^{2}\right)$ and $k_{\mu}=\sigma_{\mu} / E[\mu]$. Probability functions other than Gumbel and Lognormal may be used. (see [5])

Both $f_{k}()$ and $f_{\mu}()$ involve two parameters which are expressible in terms of expected value and standard deviation. The event that a caisson, initially at rest, will remain at rest under the action of a breaking wave force corresponds to the event that the force $k$ and the coefficient of friction $\mu$ lie within the boundaries defining the rest mode in the $k-\mu$ plane as shown in Fig. 3. Thus, the probability of a rest mode, denoted by $P_{r}$, is given by

$$
P_{r}=\int_{-\infty}^{k_{A}} f_{k}(x) d x\left[\int_{y=\mu_{0}(x)}^{\infty} f_{\mu}(y) d y\right]
$$

where $\mu_{0}()$ is given by (6) and $k_{A}$ by (7). It should be mentioned here that for a given caisson, $k_{A}$ is a function of the forcing function $P$ and is thus a random quantity. That is, $k_{A}$ in (7) is seen to be dependent on the quantities $H_{P}$ and $q=U / P=$ $\left(B * p_{U}\right) /(2 * P)$ which are random. For simplicity, the mean values of these quantities are used. The integration with respect to $y$ is

$$
\left[\int_{y=\mu_{0}(x)}^{\infty} f_{\mu}(y) d y\right]=\frac{1}{2}\left[\left(\frac{\ln \left(\mu_{0}(x)-\lambda_{\mu}\right.}{\varsigma_{\mu}}\right) / \sqrt{2}\right]
$$

A function of $x$, where $\operatorname{erfc}()$ is the complementary error function [1]. The remaining integration in (16) must be carried out numerically.

If $k$ is deterministic, say $k_{-}$, then the probability density function of $k$ is a Dirac delta function $f_{k}(x)=\delta\left(x-k_{-}\right)$and the probability of a rest mode is, from (16),

$$
P_{r}=\frac{1}{2} \operatorname{erfc}\left[\left(\frac{\ln k_{-}-\lambda_{\mu}}{\varsigma_{\mu}}\right) / \sqrt{2}\right]
$$

where $\operatorname{erfc}()$ is the complementary error function [1].

If $\mu$ is deterministic and takes on a value $\mu_{-}$, then the probability density function of $\mu$ is $f_{\mu}(y)=\delta\left(y-\mu_{-}\right)$and the probability density function of a rest mode is, from (16),

$$
P_{r}=F_{k}\left(k_{A}\right)=\exp \left[-\exp \left(-\alpha_{k}\left(k_{A}-u_{A}\right)\right)\right]
$$

provided $\mu_{-}=\mu_{A}$ in (8). Here, $F_{k}()$ is the probability distribution function of $k$ and $k_{A}$ is given in (7). If $\mu_{-}<\mu_{A}$ then $k_{A}$ in (19) should be replaced by $k_{-}^{*}$ obtained from (6) $\mu_{0}\left(k_{-}^{*}\right)=\mu_{-}=$ $k_{-}^{*} /\left(1-k_{-}^{*} q\right)$ as

$$
k_{-}^{*}=\frac{\mu_{-}}{1+q \mu_{-}}
$$

In the following, the $P_{r}$ values are calculated for the caissons in Examples 1 and 2 for the cases: (1) both $k$ and $\mu$ are random, (2) $k$ is deterministic and $\mu$ is random and (3) $k$ is random and $\mu$ is deterministic. These cases are referred to as the $G / L n$ case, the $\delta / L n$ case and the $G / \delta$ case respectively.

The expected values and standard deviation of $\mu$ are respectively chosen arbitrarily as $E[\mu]=0.6$ and $\sigma_{\mu}=0.1$; those of $k$ are $E[k]=E[P] / W$ and $\sigma_{k}=\sigma_{P} / W$, where, for Example 1, $E[P]=94.5 \mathrm{t} / \mathrm{m}^{2}$ and $\sigma_{P}=0.1 E[P]=9.45 \mathrm{t} / \mathrm{m}^{2}$. For Example 2, $E[P]=35.8 \mathrm{t} / \mathrm{m}^{2}$ and $\sigma_{P}=0.1 E[P]=3.58 \mathrm{t} / \mathrm{m}^{2}$. Other relevant quantities may be found in sections 6 and 7 and Figs. 8, 9, 10 and 11. Specifically, for Example $1, H_{P}=5.158 m, p_{U} / P=0.07$ and, for case $1(B=15.83 m), q=0.557$ and for case $2(B=19$ $m), q=0.6685$. For Example $2, H_{P}=5.183 m, p_{U} / P=0.074$ and, for case $1(B=5.667 m), q=0.209$ and for case $2, q=0.229$.

The $P_{r}$ values as well as the safety factors are all given in Table 1. These $P_{r}$ values will of course be different depending 
Table 1. Probabilities of a rest mode and safety factors for the caissons in Examples 1 and $2\left(\sigma=0.1\right.$ stands for $\sigma_{\mu}=0.1$ and $\sigma_{p}=0.1 E[P] ; \sigma=0.2$ stands for $\sigma_{\mu}=0.2$ and $\left.\sigma_{p}=0.2 E[P]\right)$.

\begin{tabular}{|c|c|c|c|c|c|c|c|c|c|c|}
\hline Example & case & $\mathrm{B}(\mathrm{m})$ & $\begin{array}{c}G / L n \\
(\sigma=0.1)\end{array}$ & $\begin{array}{c}G / L n \\
(\sigma=0.2)\end{array}$ & $\begin{array}{c}\delta / L n \\
(\sigma=0.1)\end{array}$ & $\begin{array}{c}\delta / L n \\
(\sigma=0.2)\end{array}$ & $\begin{array}{c}G / \delta \\
(\sigma=0.1)\end{array}$ & $\begin{array}{c}G / \delta \\
(\sigma=0.2)\end{array}$ & SFS & SFR \\
\hline \multirow{2}{*}{1} & 1 & 15.8 & 0.54 & 0.53 & 0.47 & 0.44 & 0.57 & 0.57 & 1.00 & 2.27 \\
\hline & 2 & 19.0 & 0.73 & 0.63 & 0.86 & 0.66 & 0.77 & 0.68 & 1.20 & 3.27 \\
\hline \multirow{2}{*}{2} & 1 & 5.68 & 0.53 & 0.50 & 0.76 & 0.59 & 0.74 & 0.66 & 1.14 & 1.00 \\
\hline & 2 & 6.22 & 0.73 & 0.60 & 0.89 & 0.69 & 0.79 & 0.70 & 1.25 & 1.20 \\
\hline
\end{tabular}

on the expected value and standard deviation of $\mu$ and $k$. Thus, by letting $\sigma_{\mu}=0.2$ and $\sigma_{P}=0.2 E[P]$, the $P_{r}$ values are recalculated and given in Table 1 as well. For brevity, these cases are identified as $\sigma=0.1$ and $\sigma=0.2$ respectively. Naturally, as the standard deviation increases, the probability of a rest mode is correspondingly reduced. In this connection, it is noted that in Example 1, for $B=15.83 \mathrm{~m}$, for the $G / \delta$ case, changing the standard deviation of $k$ does not change the $P_{r}$ value. This is due to the special choice made (arbitrarily) of the relationship between the standard deviation and the expected value: $\sigma_{P} / E[P]=$ constant.

It is of some interest to point out that in Example 1, case 1, while the safety factor against sliding is 1.00 , meaning that there is no safety margin against sliding, there is nevertheless a finite probability (around 0.5 ) that the caisson remains at rest. Similarly, in Example 2, case 1, the safety factor against rocking is 1.00 indicating that rocking is impending but the probability of a rest mode is finite.

In Example 1, case $2(B=19 \mathrm{~m})$, the safety factor against sliding is equal to 1.2 and that against rocking is 3.27 . In Example 2, case 2, the safety factor against rocking is 1.2 and that against sliding is 1.25 . The $P_{r}$ value for both cases considering the $G / L n$ case is around 0.7. It is thus seen that the safety factors against sliding and rocking are not correlated with the probability of a rest mode. This correlation can only be made if the probabilities of sliding and rocking are calculated. This can be done by first deriving the criteria corresponding to sliding and rocking. This task, though rather lengthy, may be carried out in much the same way those for a rest mode are obtained in this paper. The manner in which the probabilities of sliding and rocking are calculated is the same as demonstrated in reference [5].

\section{SUMMARY AND CONCLUSIONS}

The criteria for a caisson to be at rest under the action of a breaking wave force are derived using the equilibrium equations of the caisson together with the conditions a rest mode must satisfy. The criteria so obtained are shown to agree with those currently in use: the caisson must not slide nor rock.

The criteria are presented in the form of a graph with the applied force, $k$, normalized with respect to the weight of the caisson in water as the abscissa and the coefficient of friction as the ordinate. A region in this $k-\mu$ plane is identified as the safe region. A point that lies within the rest region indicates that the caisson will be at rest. Two examples are given to show how to use the plot to select the width of a caisson.

Since there are uncertainties associated with the applied force and the coefficient of friction, safety factors are used in current practice. The $k-\mu$ plot introduced here is a convenient way to treat $k$ and $\mu$ as random variables and assess the safety of a caisson in terms of probability. Two examples are given to show how the probability of a rest mode is calculated.

In closing, it should be mentioned that the problems related to probabilistic modeling of the random variables $k$ and $\mu$ and the assessment of the parameters that characterize these variables remain to be studied. It is mentioned here that current design practice also requires consideration of bearing capacity at the heel of the caisson [4] and much work has been done to understand the behavior of subsoil, the latest being the work of Ulker [7].

\section{ACKNOWLEDGMENTS}

The first 5 sections of this paper are taken from reference 5 .

\section{REFERENCE}

1. Abramowitz, M. and Stegun, I. A., Handbook of Mathematical Functions, Dover Publications Inc., New York, p. 1046 (1968).

2. Benjamin, J. R. and Cornell, C. A., Probability, Statistics, and Decision for Civil Engineers, McGraw Hill Inc., New York, p. 684 (1970).

3. Goda, Y., Random Seas and Design of Maritime Structures, University of Tokyo Press, Tokyo, p. 443 (1985).

4. Kuo, Y. Y. (Ed.), Complementary Study for Harbour Structure Design Standards (I), Report MOTC-IOT-97-EDB001, Institute of Transportation, Ministry of Transportation and Communications, Taichung, Taiwan, R.O.C., p. 284 (2009). (in Chinese)

5. Tung, C. C., "Criteria for initiation of motion of rigid bodies to base excitation," Earthquake Engineering and Structural Dynamics, Vol. 34, pp. 1343-1350 (2005).

6. Tung, C. C. and Lin, J. G., "On the selection of width of a caisson," Proceedings of the ASME 28th International Conference on Ocean, Offshore and Arctic Engineering, OMAE 2009, Honolulu, Hawaii, No. OMAE2009-79046 (2009).

7. Ülker, M. B. C., Dynamics of Saturated Porous Media: Wave Induced Response and Instability of Seabed, Ph.D. Dissertation, Department of Civil Engineering, North Carolina State University, Raleigh, North Carolina, U.S.A., p. 452 (2009). 